\title{
Toxicity of Chemotherapy in a Patient with Bloom Syndrome's Diagnosis
}

\author{
Candido Priscila B. M., Anjos Caroline S., Rosa Victor D. L., Rapatoni Liane and Peria Fernanda, M. \\ Division of Clinical Oncology, University Hospital, Ribeirão Preto Medical School, University of São Paulo, Ribeirão Preto \\ 14048-900, Brazil
}

\begin{abstract}
Bloom's syndrome is a rare disease that is related to an increased risk of developing malignant neoplasm. The patient reported was followed up with several hospital staff when she was diagnosed with gastric cancer, but unfortunately, she had already peritoneal implants in moment of the surgery approach. It has been reported in the literature that patients with this syndrome present greater toxicity to the chemotherapeutic treatment, however due to the rarity of the disease, it is not known exactly how the adjustment of these drugs should be performed and which is the better protocol to use. Palliative chemotherapy was proposed, but after receiving one dose of the initially treatment, she developed severe and limiting toxicity.
\end{abstract}

Key words: Bloom's syndrome, cancer, chemotherapy, toxicity.

\section{Introduction}

Bloom's syndrome is rare autosomal recessive genetic disorder caused by mutation in the BLM gene on chromosome 15 (gene locus, 15q 26.1). The first description of this syndrome was in 1954 by dermatologist David Bloom and the exact incidence is unknown [1, 2].

The gene BLM, responsible for Bloom syndrome, encodes a homologue of recQ helicase [3, 4]. Loss of helicase activity in the cells of individuals with Bloom syndrome is responsible to genomic instability, which is characterized by increased rates of somatic recombination, chromosomal breakage, and gene mutation [3, 5-7].

In 2009, there were 265 cases registered with Bloom's Syndrome. It has been reported in different ethnic groups, however, it is more common in the Jewish population (Ashkenazi) of Western Europe [8]. Patients with Bloom's syndrome have a great predisposition to the development of neoplasic diseases. By age 25, nearly 50\% of patients, unfortunately, will develop some malignancy, and many of these patients

Corresponding author: Fernanda M. Peria, Ph.D., research fields: clinical oncology. will have two or more primary neoplasms. In the first two decades of life, hematologic malignancies predominate in adult life. Among the solid tumors, malignant neoplasms of the digestive tract are most frequent. The most interesting is not the distribution of the tumors in this population, but the early age of the diagnosis when compared to general population. In general, Leukemia and lymphoma are the most commonly diagnosed malignancies followed by colorectal cancer $[9,10]$.

It has been reported in the literature that the patients with this syndrome present greater toxicity to the cytotoxic chemotherapy. However, due to the rarity of this disease, it is not known exactly how the adjustment of these drugs should be performed and which is the better protocol of treatment to use [11].

Thus, the aim of the study is to report the case of a young patient with Bloom's syndrome that developed a gastric cancer and presents a severe toxicity during the first cycle of palliative cytotoxic chemotherapy.

\section{Case Report}

A female patient started an investigation at the pediatric Outpatient Clinic at the General Hospital of 
Table 1 Features characteristics of bloom syndrome.

\begin{tabular}{|l|}
\hline Anthropometric measurements below the standard for the normal population \\
\hline Café-au-late macules throughout the body \\
\hline Diffuse hypomelanotic macules \\
\hline Bilateral fifth finger clinodactyly \\
\hline Facial features: \\
$\checkmark \quad$ Long narrow face \\
$\checkmark \quad$ Telangectasic erythema on nose, malar and oral region \\
$\checkmark \quad$ Proeminent nose \\
$\checkmark \quad$ Retrognathia \\
\hline
\end{tabular}

the University of São Paulo Medical School, Ribeirão Preto, Brazil, when she was 11 months old due to a delay in structural-weighted growth (Table 1). After almost 10 years of investigation, she was diagnosed with Bloom's syndrome by the Genetics medical team of the service.

During the years of the disease, she was followed up in several specialties because she still had the following diagnoses: erythema facial, asthma, diabetes mellitus, allergic rhinitis, and renal lithiasis.

Although the patient did not present severe complications that required hospitalization during her period of development, all these comorbidities associated with bloom syndrome were difficult to control. She underwent periodic exams and, despite all these care, she complained of postprandial epigastric pain. The investigation began with an ultrasound of the abdomen, but the result did not show any alterations that justified the patient's complaints. After that, it took several months for the patient to perform the necessary tests, due to socioeconomic problems; the patient did not appear for scheduling the procedure. After at least 3 attempts, she was submitted to upper digestive endoscopy and was diagnosed with gastric adenocarcinoma at 21 years old.

After the diagnosis, the patient did stage exams with tomography of the abdomen and thorax and as the exams did not show distant metastasis, the patient underwent total gastrectomy. Unfortunately, during the surgical procedure it was evidenced the presence of peritoneal carcinomatosis characterizing clinical stage IV of the disease.

Due to Bloom's syndrome, the young woman weighed only $21 \mathrm{~kg}$ and was $130 \mathrm{~cm}$ tall. Palliative chemotherapy was proposed with Xelox protocol (Oxaliplatin 130mg/m2 on D1 and Capecitabine $2000 \mathrm{mg} / \mathrm{m} 2$ on D2-D15). In the first cycle, after receiving one of the proposed drugs (oxaliplatin), she developed severe bronchospasm immediately after the drug infusion and hyperglycemia. At that time, it was decided to not release the capecitabine tablets because the medical team judged the risk of other toxicities was very high. Thus, even receiving only one drug, by the 10th day after chemotherapy, the patient presented nausea, vomiting, diarrhea and pancytopenia. She had to remain hospitalized for approximately three weeks to control the symptoms and had to receive intravenous antibiotics, as she was still suffering from febrile neutropenia.

The symptoms were partially controlled and an outpatient follow-up was proposed. The chemotherapy treatment had to be permanently suspended and the patient died almost 2 months later due to acute obstructive abdomen secondary to disease progression.

\section{Discussion}

Bloom syndrome should be suspected in patients with early onset neoplasm, short stature and photosensitivity on the face. The diagnosis can be confirmed by cytogenetic evaluation and the most significant implication of a diagnosis is the high risk of developing cancer [11, 12].

The clinical case describes the trajectory of a patient who confirmed the diagnosis of Bloom Syndrome by the age of 11 years. The young woman presented several of the comorbidities associated with this 
autosomal recessive disease, necessitating, therefore, the monitoring of several specialties of the service. Even so, the control of symptoms, respiratory and hyperglycemia related to insulin resistance, were the most difficult to control.

It is a rare disease and therefore there is no approved guideline for the management of these individuals. This could explain why even the patient performing follow-up with different specialties within same service, the diagnosis of cancer was made late.

Cancer is the most serious and frequent complication of patients with this syndrome and, unlike other heritable syndromes, which the possibility of developing this disease is related to a small variety of tumors [3, 9, 10]. In Bloom syndrome there are reports in the literature of numerous types of primary neoplasms. Thus, the lack of evidence to propose a standardized screening aiming at an early diagnosis of so many tumors make cancer the main cause of death in this group of individuals.

Among the solid tumors, the neoplasms of the digestive tract are the most frequent, mainly adenocarcinoma digestive tract upper and lower. Being the first, the diagnosis of the patient was described in this clinical case.

Many reports indicate that patients with Bloom's syndrome have a greater sensitivity to treatment of malignancy and consequently increase toxicity. German registered severe treatment reactions in patients with acute leukemia, including myelotoxicity, pneumonitis, hepatitis, intestinal hemorrhage and mucositis despite the doses reduces of chemotherapy [9]. Other authors reported a case of esophageal stricture due to radiotherapy for lung cancer [13].

As mentioned in the literature, toxicity secondary to chemotherapy drew attention in this clinical case and limited the continuity of treatment. The intensity of the adverse effects presented by the patient was not expected for people of the same age and sex. The young woman received only one of the drugs of the proposed palliative treatment and required medical care in a hospital setting.

\section{Conclusions}

It is known that patients with Bloom's syndrome are an increased risk of early developing solid and hematological tumors. The risk increases with increasing age, but there is no evidence that the screening is benefit to these patients.

According to the severe toxicity caused by chemotherapy in patients with this syndrome, it is required to be caution in the choice of chemotherapeutic agents and the dose adjustment during the treatment.

We report a case of a Bloom syndrome patient with metastatic gastric cancer who developed several toxicities with cytotoxic chemotherapy early in the first cycle, suggesting that the molecular alterations as the genomic instability leads to hypersensitivity to the treatment causing striking greater side effects.

\section{References}

[1] Bloom, D. 1954. "Congenital Telangiectatic Erythema Resembling Lupus Erythematosus in Dwarfs.” Am. J. Dis. Child. 88: 754.

[2] Ellis, N. A., and German, J. 1996. "Molecular Genetics of Bloom's Syndrome.” Hum. Molec. Genet. 5: 1457-63.

[3] German, J. 1993. "Bloom Syndrome: a Mendelian Prototype of Somatic Mutational Disease.” Medicine (Baltimore) 72: 393-406.

[4] Ellis, N. A., Groden, J., Ye, T. Z., Straughen, J., Lennon, D. J., Ciocci, S., et al. 1995. "The Bloom's Syndrome Gene Product is Homologous to RecQ Helicases.” Cell 83: 655-66.

[5] Hickson, I. D. 2003. "RecQ Helicases: Caretakers of the Genome.” Nat Rev Cancer 3:169-177.

[6] Groden, J., and German, J. 1992. "Bloom's Syndrome. XVIII. Hypermutability at a Tandem-repeat Locus.” Hum. Genet. 90: 360-7.

[7] Groden, J., Nakamura, Y., and German, J. 1990. "Molecular Evidence that Homologous Recombination Occurs in Proliferating Human Somatic Cells.” Proc. Natl. Acad. Sci. 87: 4315-9.

[8] German, J., Bloom, D., Passarge, E., Fried, K., GOodman, R. M., Katzenellenbogen, I., et al. 1997. "Bloom's Syndrome VI: The Disorder in Israel and an Estimation of the Gene Frequency in the Ashkenazim.” Am. J. Hum. 
Genet. 29: 553-62.

[9] German, J. 1992. Bloom's Syndrome: Incidence, Age of Onset, and Types of Leukaemia in the Bloom's Syndrome Registry. Washington DC.

[10] Sanz, M. M., German, J., and Cunniff, C. 2006. “Bloom’s Syndrome” In GeneReviews ${ }^{\circledR}$, edited by Pagon, R. A., Adam, M. P., and Ardinger, H. H.

[11] Thomas, E. R., Shanley, S., Walker, L., and Eeles, R. 2008. "Surveillance and Treatment of Malignancy in Bloom
Syndrome.” Clin Oncol 20 (5): 375-9.

[12] Masmoudi, A., Marrakchi, S., Kamoun, H., Chaaben, H., Ben Salah, G., Ben Salah, R., et al. 2012. "Clinical and Laboratory Findings in 8 Patients with Bloom's Syndrome.” J Dermatol Case Rep 1: 29-33.

[13] Kataoka, M., Kawamura, M., Hamamoto, K., Takemiya, M., and Miki, Y. 1989. "Radiation-induced Oesophageal Stricture in a Case of Bloom's Syndrome.” Clin oncol 1: 47-8. 